\title{
Citation:
}

Madison, A.A., Shrout, M.R., Renna, M.E., Kiecolt-Glaser, J.K. (in press) Psychological and Behavioral Predictors of Vaccine Efficacy: Considerations for COVID-19, Perspectives on Psychological Science.

\section{Psychological and Behavioral Predictors of Vaccine Efficacy: Considerations for COVID-19}

\author{
Annelise A. Madison ${ }^{1,2}$, M. Rosie Shrout ${ }^{1}$, Megan E. Renna ${ }^{1,3}$, Janice K. Kiecolt-Glaser ${ }^{1,4}$
}

${ }^{1}$ The Institute for Behavioral Medicine Research, The Ohio State University College of Medicine

2Department of Psychology, The Ohio State University

${ }^{3}$ The Comprehensive Cancer Center, The Ohio State University College of Medicine

${ }^{4}$ Department of Psychiatry and Behavioral Health, The Ohio State University College of Medicine

Corresponding author: Janice Kiecolt-Glaser, Institute for Behavioral Medicine Research, The Ohio State University College of Medicine, 460 Medical Center Drive, Columbus, $\mathrm{OH} 43210$, 614-293-3499 (telephone), 614-366-2097 (fax), Janice.Kiecolt-Glaser@osumc.edu.

Work on this paper was supported in part by NIH grants TL1TR002735, R01 AG069138, R01 AG057032, R01 CA186251, R01 CA186720, and T32CA229114. The content is solely the responsibility of the authors and does not necessarily represent the official views of the NIH.

Short title: Psychological Predictors of Vaccine Response 


\begin{abstract}
Severe acute respiratory syndrome coronavirus 2 (SARS-COV-2) vaccine candidates are being evaluated, with the goal of conferring immunity on the highest percentage of people who receive the vaccine as possible. Importantly, this percentage, termed vaccine efficacy, depends not only on the vaccine, but also on characteristics of the vaccinated. Over the past 30 years, a series of studies has documented the impact of psychological factors on the immune system's vaccine response. Robust evidence has demonstrated that stress, depression, Ioneliness, and poor health behaviors can impair the immune system's response to vaccines, and this effect may be greatest in vulnerable groups like the elderly. Psychological factors are also implicated in the prevalence and severity of vaccine-related side effects. These findings have generalized across many vaccine types, and therefore may be relevant to the SARS-COV2 vaccine. In this review, we discuss these psychological and behavioral risk factors for poor vaccine responses, their relevance to the COVID-19 pandemic, as well as targeted psychological and behavioral interventions to boost vaccine efficacy and reduce side effects. Recent data suggest these psychological and behavioral risk factors are highly prevalent during the COVID-19 pandemic, but intervention research suggests that psychological and behavioral interventions can increase vaccine efficacy.
\end{abstract}




\section{Introduction}

Even the best vaccines do not work for everyone. Some vaccines, like the measles vaccine, are highly efficacious, reducing infection rates by about $98 \%$. The U.S. Food and Drug Administration said that it will approve any severe acute respiratory syndrome coronavirus 2 (SARS-CoV-2) vaccine that prevents the coronavirus-19 disease (COVID-19) in at least $50 \%$ of those who receive it (i.e., $50 \%$ efficacy), a relatively low bar. Initial reports suggest that the Pfizer BioNTech and Moderna vaccines are 95\% efficacious (e.g., Mahase, 2020), which is highly impressive given the condensed timeline for vaccine development. However, the AstraZenica vaccine is less efficacious, with only $62 \%$ efficacy among those given two full vaccine doses and $90 \%$ in the subset given a half dose followed by a full one (Knoll \& Wonodi, 2020). Effectiveness, or how much the vaccine reduces infection rates in the "real world" outside of the highly-controlled clinical trial setting-, is often lower than the initial efficacy rate. Moreover, these reported efficacy rates are based on a relatively short follow-up period, and it is unknown how the vaccine will perform over time. Although vaccine efficacy depends heavily on vaccine-related factors, characteristics of the vaccinated also matter. This review focuses on the latter, as some of these characteristics like stress are rampant during the pandemic and can not only reduce vaccine efficacy but also promote more immediate and transient side effects such as fatigue and low mood; however, many of these factors are modifiable and thus may be important intervention targets as the world prepares for widespread immunization.

Psychological, social, and behavioral factors can substantially impact the immune system's vaccine response. Our lab spearheaded this line of research in the early 1990s with the initial observation that psychological factors shaped even young and healthy people's antibody responses to vaccines (Glaser et al., 1992). Since then, a plethora of studies have helped to clarify the psychological factors and poor health behaviors that increase the risk for 
vaccine non-responsiveness. We will review these findings and discuss their relevance to the COVID-19 pandemic, following a brief summary of the immune system's multifaceted response to vaccines.

\section{The Immune System's Immediate and Delayed Vaccine Response}

All vaccines challenge the immune system. Inflammatory markers rise within hours of vaccination - thanks to the immediate and non-specific innate immune response, which can produce side effects like lethargy, malaise, and irritability. As the first prong of the immune response, the inflammatory response usually lasts a few days but can be prolonged in some individuals, such as those who are depressed (Glaser, Robles, Sheridan, Malarkey, \& KiecoltGlaser, 2003). The adaptive immune system mounts the second prong of the immune response. It targets unique vaccine components and therefore takes longer to launch. Vaccines are designed to give the adaptive immune system a lasting memory of viral or bacterial components, so that it can quickly and effectively respond when confronted with the actual pathogens. The adaptive immune system responds to the vaccine through (1) T cell multiplication, which can be programmed to identify and kill cells that contain the pathogen (i.e., the cell-mediated response), and (2) B cell production of antibodies, or proteins that neutralize viruses and bacteria.

One critical factor that modulates this response is whether the vaccine recipient has previously encountered the antigen, or protein on the surface of pathogen - either via infection or vaccination. If so, the body mounts a faster and fiercer antibody response - the secondary immune response - than it did during the first encounter (i.e., primary immune response). One limitation of this literature is that some studies do not fully account for prior exposure, making it difficult to decipher whether the primary or secondary immune response is reported (Cohen, Miller, \& Rabin, 2001). However, failure to account for prior exposure can mask the magnitude of the impact of stress. Especially among older adult samples, it is often safe to assume that they have already encountered certain antigens and therefore mount a secondary immune 
response (Cohen et al., 2001). This is a key consideration for the SARS-COV-2 vaccine, as around 10\% of Americans had prior exposure as of September 2020 (Bajema et al., 2020); many more have had exposure to other coronaviruses, which may influence immune responses to SARS-COV-2 (Poland, Ovsyannikova, \& Kennedy, 2020); and some of the current vaccine candidates require multiple doses.

The studies we will review typically report the antibody, rather than cellular, response, but as stated above, antibody release is just one facet of the adaptive immune system's response. Because the SARS-COV-2 virus is novel, it is not yet known how different immune cells and antibodies protect against infection. Cell-mediated (T cell) immunity may play an important role in preventing COVID-19 reinfection because antibody levels naturally wane months after infection (Dan et al., 2020). Even so, there is little evidence that people have robust cell-mediated immunity in the absence of an antibody response, and in one pre-print still undergoing peer review, the peak antibody response aligned with the T cell response. Thus, antibody titers (levels) may be an important early indicator of lasting immunity (Zuo et al., 2020). Indeed, the antibody titer is considered to be a clinically significant biomarker of protection against SARS-COV-2 (Poland et al., 2020), although it is not the only such biomarker. It is wellestablished that COVID-19 patients have a highly heterogenous antibody response with greater disease severity associated with more antibody, which itself predicts clinical outcomes (W. Tan et al., 2020). Antibody levels remain highly disparate months later: one study reported a 200fold difference in SARS-COV-2 antibody levels six months after infection (Zuo et al., 2020) important variability that may map on to patient characteristics.

\section{Stress, Depression, and Other Psychological Factors}

Prior to our lab's vaccine studies, there was already evidence that stress impacted various aspects of immune function, but the clinical relevance was unclear. Vaccination is not only beneficial for participants and directly applicable to clinical settings, it is also a helpful paradigm to assess the immune system's ability to respond to pathogens, as everyone receives 
the same standardized dose but responses can vary widely. In our first study, we administered the standard series of three hepatitis B inoculations over six months to medical students, each on the third day of three-day of stressful academic examination blocks (Glaser et al., 1992). After the first dose, $25 \%$ of the students developed an antibody response to hepatitis B. These early responders had lower perceived stress and anxiety than those who responded later. Intriguingly, earlier and later responders' stress, anxiety, and social support had not differed across the earlier academic year, suggesting that their divergent vaccine responses were related to the more proximal stressful examination period. One year later, these findings were replicated (Jabaaij et al., 1993). Subsequent research among healthy young adults revealed that self-rated stress levels in the ten days after vaccination may be more influential for the antibody response than stress in the prior two days, and stress-related sleep loss may be a primary culprit (Miller et al., 2004).

We expanded on this work by looking at vaccine responses in older adults, as stressrelated immune dysregulation is most pronounced among the elderly (Glaser \& Kiecolt-Glaser, 2005). The weakened aging immune system is preoccupied with keeping at bay a whole host of pathogens accumulated throughout the lifetime (Franceschi et al., 2000), so it is less responsive to new immune challenges like vaccines. We first compared the influenza virus vaccine responses of long-term dementia spousal caregivers and age-, sex-, and socioeconomicallymatched non-caregivers (Kiecolt-Glaser, Glaser, Gravenstein, Malarkey, \& Sheridan, 1996). Caregiving, especially for someone with dementia who requires more intensive and round-theclock care, is a chronic stressor, often leading to a reduced social network, disengagement from hobbies, and increased risk for anxiety and depressive disorders. Within four weeks, only $38 \%$ of caregivers had a clinically significant antibody response to the vaccine - defined as a fourfold increase in antibody titers (levels) within four weeks - whereas $66 \%$ of non-caregivers responded, and older age accentuated these differences. In another study caregivers' higher cortisol levels helped to explain this disparity (Vedhara et al., 1999). Our caregivers also had 
poorer cell-mediated vaccine responses, which may be more consequential for older adults' immune functioning than antibody responses (Lang et al., 2010). Our findings from 1996 are particularly noteworthy because we only included participants who had received an influenza virus vaccine the previous year, which can influence the antibody response to the current vaccine as certain components of the vaccine are often repeated from one year to the next, and there is cross-reactivity among some influenza strains. Also, we found that caregiving status was associated with both major facets of the adaptive immune response: antibodies and T cells. Subsequent studies in our lab revealed that caregivers' weak immune response to the influenza virus vaccine persisted long after their spouses' death (Glaser, Kiecolt-Glaser, Malarkey, \& Sheridan, 1998).

We then investigated whether these findings among caregivers would generalize to a pneumococcal pneumonia vaccine, which protects against a bacterial infection, rather than a virus. Of note, the immune system's response to this type of vaccine is T cell independent and therefore no memory B cells are produced, which means that the body launches a primary immune response even upon repeated exposure. Current and former caregivers and controls did not have different antibody responses two weeks or one month post-vaccination, but current caregivers were unable to maintain their antibody levels, with a relative decline three and six months post-vaccination (Glaser, Sheridan, Malarkey, MacCallum, \& Kiecolt-Glaser, 2000). Thus, caregiving stress ultimately eroded the initial vaccine antibody response, perhaps rendering caregivers more susceptible to infection. Similar findings among younger adults also indicate that psychological factors like stress and social support shape immune responses to bacterial vaccines (Gallagher, Phillips, Drayson, \& Carroll, 2009b; Gallagher, Phillips, Ferraro, Drayson, \& Carroll, 2008).

Even younger caregivers have poorer antibody responses to vaccines. Parental caregivers of children with developmental disabilities had lower antibody responses to the influenza virus vaccine, adjusting for baseline antibody levels, (Gallagher, Phillips, Drayson, \& 
Carroll, 2009a) and pneumococcal pneumonia vaccine (Gallagher et al., 2009b), which evokes a primary immune response, compared to parents of typically developing children. These findings indicate that intensive caregiving - even among young people - can increase susceptibility to both bacterial (Gallagher et al., 2009b; Glaser et al., 2000) and viral (Gallagher et al., 2009a; Glaser et al., 1998; Kiecolt-Glaser et al., 1996) infections.

Among caregivers, psychological distress and negative thought patterns may influence vaccine responsiveness. Spouses and children of community-dwelling Alzheimer's disease patients who had greater perceived stress and depressive symptoms had smaller antibody increases following a tetanus vaccine, even after adjusting for baseline antibody levels, which were already providing clinical protection for most of these caregivers (Li et al., 2007). Similarly, among caregivers, negative repetitive thought predicted greater post-vaccination depression and lower antibody titers, again controlling for baseline titers (Segerstrom, Schipper, \& Greenberg, 2008).

In sum, across different vaccine types, both long-term stressors like caregiving and short-term stressors like an academic examination can impair vaccine responses — particularly the antibody response, which is a primary endpoint in many studies, but also there is some evidence that the cell-mediated response is also weaker (e.g., Glaser et al., 1992; KiecoltGlaser et al., 1996). However, it is notable that very brief (e.g., 10 minute) stressors with a clear endpoint that occur immediately post-vaccination can ultimately enhance antibody responses (e.g., Brydon, Walker, Wawrzyniak, Chart, \& Steptoe, 2009) but may trigger side effects, as discussed below. Distressed individuals' poorer vaccine responses can persist long after the vaccine administration (e.g., Glaser et al., 2000) and long after the end of the stressor (e.g., Glaser et al., 1998). In particular, our finding that caregivers' primary antibody responses were not initially lower two weeks and one month after a pneumococcal pneumonia vaccine but then declined relative to non-caregivers three and six months post-vaccination (Glaser et al., 2000) may be particularly relevant to the SARS-COV-2 vaccine because it 1) showed that stress can 
affect the primary immune response, and 2) suggests that stress may erode antibody levels over time. For a significant subset of the population, the SARS-COV-2 vaccine will be the first time they encounter the antigen, in which case the primary immune response is relevant. Also, it is not known how long the SARS-COV-2 vaccine candidates will protect recipients from infection. Although the current leading vaccine candidates have generally achieved high efficacy, it is possible that vaccine recipients' chronic stress may lessen this response over time, thus necessitating more frequent vaccination to maintain immunity.

Another line of evidence shows that both state and trait psychological factors may impact the shorter-term innate immune response to vaccination, thereby helping to determine the number and severity of post-vaccination side effects people experience. When it comes to trait psychological factors and chronic stress, the research is primarily correlational (e.g., Glaser et al., 2003). However, there is compelling experimental evidence about acute stress from a double-blind randomized, placebo-controlled trial in which participants were assigned to one of four conditions: typhoid vaccine/rest, placebo vaccine/rest, typhoid vaccine/stress, placebo vaccine/stress (Brydon, Walker, Wawrzyniak, Whitehead, et al., 2009). Like the pneumococcal pneumonia vaccine, the typhoid vaccine also triggers a primary immune response regardless of prior exposure. Upon vaccination, participants either rested or completed 10 minutes of mentally challenging tasks - a Stroop task and a speech task. Even this brief stressful period amplified the inflammatory response to the vaccine, and participants had a larger increase in negative mood after the stressor if they had received the typhoid vaccine rather than the placebo (Brydon, Walker, Wawrzyniak, Whitehead, et al., 2009), demonstrating that stress and vaccination can have synergistic effects. The same lab later reported that among those with high levels of trait optimism, these effects were buffered (Brydon, Walker, Wawrzyniak, Chart, et al., 2009). Thus, the interplay between state and trait psychological factors may contribute to post-vaccination side effects. 
Depression alters multiple facets of the vaccine responses. Many depressed individuals' immune systems are dysregulated even prior to receiving a vaccine, as evidenced by heightened levels of inflammation (Kiecolt-Glaser, Derry, \& Fagundes, 2015). This chronic inflammation may interfere with the vaccine response (Vukmanovic-Stejic et al., 2018). Indeed, unmedicated depressed patients - all with prior exposure to varicella zoster - had lower cellmediated responses to a varicella zoster virus vaccine than both depressed individuals on antidepressants and non-depressed individuals, suggesting that they may be at an increased risk for a herpes zoster recurrence, i.e., shingles (Irwin et al., 2013). Among hemodialysis patients, those with more depressive symptoms had a lower antibody response to the hepatitis B vaccine (Afsar, Elsurer, Eyileten, Yilmaz, \& Caglar, 2009). Adding fuel to the fire, depressive symptoms may amplify and prolong the acute inflammatory response to a vaccine (Glaser et al., 2003). Chronically elevated inflammation reduces the body's ability to fight infections, and it also accelerates the aging of the immune system, a process called "inflamm-aging" (Franceschi et al., 2000).

Other psychological factors that predicted lower antibody responses to vaccination included high trait negative affect (Marsland, Cohen, Rabin, \& Manuck, 2001), low trait positive affect (Marsland, Cohen, Rabin, \& Manuck, 2006), high neuroticism (Morag, Morag, Reichenberg, Lerer, \& Yirmiya, 1999; Phillips, Carroll, Burns, \& Drayson, 2005), and low selfesteem (Morag et al., 1999). Not surprisingly, these dispositional factors increase distress as well as risk for depression.

\section{Psychological Health During the COVID-19 Pandemic}

These studies from our lab and others have demonstrated how depression and psychological stress - even just a few days before or after the vaccine - can be a powerful and robust predictor of the immune system's innate and adaptive response. Unfortunately, distress is integral to the COVID-19 pandemic; in fact in one U.S. sample, the fear of COVID-19 itself, termed "coronaphobia," drove depression and generalized anxiety, even after adjusting for 
sociodemographic factors and other psychological vulnerability factors like neuroticism (S. A. Lee, Jobe, Mathis, \& Gibbons, 2020). In another large representative U.S. sample, those with elevated COVID-19 fearfulness were at a particularly high risk for clinically significant depressive symptoms (Fitzpatrick, Harris, \& Drawve, 2020). Ironically, fear of COVID-19 itself may lessen a vaccine's ability to confer immunity against the virus.

The prevalence of psychiatric symptoms and clinical diagnoses have increased during the worldwide pandemic. According to the U.S. Census Bureau, adults in April and May 2020 had triple the likelihood of screening positive for either a depressive disorder, anxiety disorder, or both, compared to adults surveyed in early 2019; in fact, during the pandemic, one in three U.S. adults screened positive for one or both disorder types (Twenge \& Joiner, 2020). Between April and May 2020, the prevalence of anxiety declined while depression rose (Twenge \& Joiner, 2020). In a large representative U.S. sample, the average depressive symptom score was almost one point above the cut score used to identify clinically significant depressive symptoms, reflecting widespread distress (Fitzpatrick et al., 2020). Additionally, those who reported food insecurity were particularly at risk (Fitzpatrick et al., 2020), which is concerning given that low socioeconomic status is related to COVID-19 severity (Raifman \& Raifman, 2020). Similarly, during the COVID-19 outbreak in China in February 2020, an online survey found that prevalence rates for clinically significant generalized anxiety symptoms, depressive symptoms, and poor sleep quality among the general population were $35 \%, 20 \%$, and $18 \%$, respectively (Huang \& Zhao, 2020). This research suggests that elevated stress, depression, and anxiety are more prevalent during the COVID-19 pandemic, and certain demographics, such as those who are more fearful of COVID-19 or those with lower socioeconomic status, are particularly likely to experience these symptoms - as well as reduced vaccine efficacy.

The above research also demonstrates that both state and trait psychological factors may help determine the prevalence and severity of vaccine-related side effects. For example, experiencing an acute stressful event immediate after vaccination may worsen side effects 
(Brydon, Walker, Wawrzyniak, Whitehead, et al., 2009). The possibility of SARS-COV-2 vaccine-related side effects is one factor determining U.S. adults' willingness to be vaccinated (Reiter, Pennell, \& Katz, 2020). To whatever extent possible, reducing stress exposure around the time of vaccination may help to reduce the likelihood of bothersome side effects (Brydon, Walker, Wawrzyniak, Whitehead, et al., 2009).

\section{Loneliness and Social Support}

Like stress, loneliness can impair immune function, even altering young and healthy peoples' vaccine responses. In one study among undergraduate students, social dysfunction predicted lower antibody levels following a meningitis C conjugate vaccine (Burns, Drayson, Ring, \& Carroll, 2002). Another study found that lonelier undergraduate students in the first semester of their first year had lower antibody levels one and four months after their first ever influenza vaccination, and a small social network (i.e., 4-12 people contacted in the past month) magnified the inadequate response (Pressman et al., 2005). Lonelier individuals did not have a poorer antibody response when their social network size was large (19-20 members), suggesting that contact with many people may provide some protection even if it is not subjectively satisfying (Pressman et al., 2005). Moreover, those who were lonely felt more overwhelmed and stressed, which contributed to their weaker antibody responses (Pressman et al., 2005), demonstrating that the elevated stress levels of those without an adequate social buffer has immune consequences. Social support also played a central role in our first vaccine study (Glaser et al., 1992): Although self-reported support was initially unrelated to total immune response (i.e., a T cell and B cell summary score), it explained $13 \%$ of the variance in total immune response after the third hepatitis $B$ inoculation - a better predictor than even their immune function a few months earlier (Glaser et al., 1992).

Social networks contract with age, as older adults prioritize relationships that are the most meaningful (Carstensen, 1992). Therefore, emotional closeness with significant others increases, and a smaller social network does not necessarily foster loneliness (Carstensen, 
1992). In fact, subjective well-being rises in later life (Jivraj, Nazroo, Vanhoutte, \& Chandola, 2014). Vaccine responsiveness may reflect this age-related shift in social goals. In an elderly sample, bereavement in the year before vaccination predicted a poorer antibody response, while those who were married and had high marital satisfaction had a stronger antibody response (Phillips et al., 2006). These findings demonstrate the differential impact of various interpersonal stressors throughout the lifespan, suggesting that social network size is especially relevant for younger adults, while loss of a spouse may be more immunologically relevant (and common) for older individuals.

\section{Loneliness and Social Support During the COVID-19 Pandemic}

During the pandemic, physical distancing measures reduced face-to-face contacts. The social networks of a cohort of students in Switzerland assessed during the pandemic were smaller than a pre-pandemic cohort, which predicted worsening mental health trajectories (Elmer, Mepham, \& Stadtfeld, 2020). Beyond social network size, relationship depth and quality also matter. Drawing on existing relationship theory and research, a recent conceptual paper predicted that COVID-19-related stress would amplify destructive dyadic processes, such as hostility and withdrawal, and that certain pre-existing contextual factors (e.g., social status, age, mental health) could buffer or bolster this link (Pietromonaco \& Overall, 2020), but further empirical work is needed. Zooming out from romantic relationships, recent data show high and increasing rates of loneliness during the U.S. outbreak (Killgore, Cloonan, Taylor, Miller, \& Dailey, 2020) - although another U.S. study failed to find large increases in loneliness (Luchetti et al., 2020). Overall, the number of face-to-face contacts has decreased during the pandemic, but it is possible that the quality and depth of specific relationships have improved. Even so, individuals who are lonely or socially isolated remain at risk for insufficient vaccine responses that do not confer immunity.

\section{Health Behaviors Matter}


Stressed individuals often have poor health behaviors, such as smoking, a low-quality diet, poor sleep habits, sedentariness, and alcohol overuse. At more extreme levels, health behaviors may have direct associations with vaccine responses, or may synergistically interact with stress to predict vaccine response (e.g., Segerstrom, Hardy, Evans, \& Greenberg, 2012).

\section{Cigarette Smoking}

Smoking depresses the antibody response to hepatitis B vaccination, as shown in multiple older studies (e.g., Struve et al., 1992; Winter, Follett, Mclntyre, Stewart, \& Symington, 1994). In fact, a recent meta-analysis found that smokers had 1.53 times the risk of nonresponse to the hepatitis B vaccine, compared to non-smokers (Yang et al., 2016). Chronic inflammation may link smoking with a poorer vaccine response (Younas, Carrat, Desaint, Launay, \& Corbeau, 2017).

\section{Nutrition}

Dietary components and nutritional status are relatively unexplored in relation to vaccine responses among healthy adults. Much of the research in this domain centers on populations in which undernourishment is common: children in developing countries and older adults. Malnourished children are generally able to mount a sufficiently protective immune response upon vaccination, but the extent and duration may be less than ideal (Prendergast, 2015). Across several studies, deficiencies in protein, vitamins A and D, iron, and zinc had little to no effect on vaccine response in children (Savy et al., 2009).

Although a single nutrient or nutrient deficiency may have little impact on vaccine response, overall diet may be an important consideration (Butler \& Barrientos, 2020). For instance, the Western Diet, high in fat, refined sugars, and processed foods, is responsible for an epidemic of chronic inflammation and obesity (Christ, Lauterbach, \& Latz, 2019). Inflammation is higher among the obese, in part because fat cells can themselves increase inflammatory signaling, which reduces the immune system's ability to mount an effective response to subsequent immune challenges (Park et al., 2014). Additionally, diet powerfully 
shapes the gut microbiota (David et al., 2014), and the gut microbiota also determines vaccine responses (Harris et al., 2018; Oh et al., 2014). As a primary example, dietary fiber intake promotes a greater abundance of bacteria like Bifidobacteria that produce short-chain fatty acids, which can boost antibody responses (Huda et al., 2014; Lynn \& Pulendran, 2018).

\section{Sleep}

Sleep substantially impacts immune function. Those who are regularly sleep deprived are not only at great risk for vaccine non-responsiveness, but also for severe illness. The relationship between disturbed sleep and lower antibody responses has been documented in both cross-sectional studies (Burns et al., 2002) and research with experimentally induced sleep restriction (Spiegel, Sheridan, \& Van Cauter, 2002). For the latter, healthy young men who normally spent between 7.5 and 8.5 hours in bed were restricted to 4 hours per night in bed for six nights, which then lengthened to 12 hours per night for 7 nights to recover from the deprivation. On the morning after the fourth short night of sleep, they received an influenza virus vaccine. Despite the period of sleep recovery, these individuals had lower antibody production than their normally-rested peers 10 days post-vaccination, even accounting for baseline antibody titers. The notable between-subject variation within each group suggested that sleep deprivation does not uniformly impair antibody responses. By three to four weeks after vaccination, their antibody levels no longer differed from their peers (Spiegel et al., 2002).

In another sleep restriction study, young adults who were allowed a normal night's sleep after a hepatitis $A$ vaccine had double the antibody response one month later, compared to those who were not allowed to sleep for 36 hours post-vaccination (Lange, Perras, Fehm, \& Born, 2003). The enhanced antibody response mirrored the normal-sleepers' increased release of immune-stimulating hormones the night and day after vaccination, and their lower levels of stress hormones. Importantly, none of these participants had been infected with hepatitis A and had very low antibody titers prior to vaccination, suggesting that sleep deprivation modulates the primary immune response. The same research group later used a similar sleep restriction 
paradigm with a hepatitis A vaccination and found marked differences in cell-mediated adaptive immunity - effects that were still evident one year post-vaccination -, and concluded that sleep fosters improved immune memory (Lange, Dimitrov, Bollinger, Diekelmann, \& Born, 2011).

Among midlife adults, sleep duration also matters for vaccine efficacy. In one study, among participants who had no serological evidence of prior hepatitis B exposure, those who reported less sleep - especially on the two nights before a hepatitis B vaccination - had lower antibody titers one and four months later (Prather et al., 2012). Similarly, sleep duration, measured objectively via actigraphy and averaged the three nights before and three nights after the first hepatitis B inoculation, predicted subsequent antibody responses and clinical protection status after the second and third shots (Prather et al., 2012). In fact, each additional hour of sleep tracked with a $56 \%$ increase in antibody levels. Taken together, this evidence suggests that shorter sleep duration lowers antibody responses - at least initially (e.g., Spiegel et al., 2002)- and fosters longer-lasting deficiencies in cell-mediated immunity across a variety of vaccines and regardless of prior exposure (Lange et al., 2011, 2003; Prather et al., 2012; Spiegel et al., 2002).

\section{Sedentariness and Physical Activity}

Physical activity promotes a strong immune system and better vaccine responses. Physically fit elderly had better antibody, but not cell-mediated, immune responses to tetanus and influenza virus vaccines, compared to their less-fit peers (Keylock et al., 2007).

Accelerometer data collected from elderly Singaporean Chinese women showed that those who walked more (>18,509 steps/day) for two weeks after an influenza virus vaccination had greater innate immune activation two days post-vaccine, larger adaptive immune responses one week post-vaccination, and greater antibody responses after a second vaccination than their less active peers ( $<10,927$ steps per day) (Choon Lim Wong et al., 2019). Similarly, physically active older men, who had done regular aerobic exercise at least three times per week for at least two years, had higher antibody responses to a novel immune challenge, compared to their peers 
who had not regularly exercised for at least the past two years (Smith, Kennedy, \& Fleshner, 2004). Adults aged 62 years and older who engaged in at least 20 minutes of vigorous exercise (i.e., intense enough to cause large increases in heart rate, breathing, and sweating that makes it somewhat difficult to have a conversation) three or more times per week had greater antibody and cellular responses to an influenza virus vaccine compared to moderately active or sedentary older adults (Kohut, Cooper, Nickolaus, Russell, \& Cunnick, 2002). These results implicate physical activity in vaccine responses to both novel (Smith et al., 2004) and familiar (Choon Lim Wong et al., 2019; Kohut et al., 2002) antigens.

\section{Alcohol Consumption}

Moderate alcohol use may not weaken the immune response to a vaccine. In fact, moderate use may even upregulate responses: rhesus monkeys that had a moderate alcohol intake for seven months had enhanced antibody and cell-mediated response to a booster shot, but those who chronically consumed high levels of alcohol had weaker responses (Messaoudi et al., 2013). Research among humans similarly suggests that moderate alcohol use bolsters immune function, as a greater number of alcoholic drinks (up to three or four per day) is associated with reduced risk of developing a cold upon exposure to the virus (Cohen, Tyrrell, Russell, Jarvis, \& Smith, 1993). However, alcohol overuse and abuse clearly harms immune function: Half of those who had chronically high alcohol intake and liver cirrhosis did not have any detectable antibody titers to hepatitis B after receiving 3-4 doses (Degos et al., 1986). All of those without cirrhosis did have a detectable but relatively low antibody response (Degos et al., 1986). Thus, the relationship between alcohol use and immune response to vaccine may be an upside-down U-shaped curve, with better responses up to a certain level of alcohol use and then diminishing responses past that point.

\section{Health Behaviors During the COVID-19 Pandemic}

The COVID-19 pandemic is undermining health behaviors. A recent commentary called attention to the well-established link between stress and problematic drinking behavior, 
suggesting that the pandemic will result in a spike in substance addiction that would further strain treatment and rehabilitation services (Clay \& Parker, 2020). Indeed, data suggest that alcohol use is more prevalent during the COVID-19 pandemic. For instance, U.S. alcohol sales increased 54\% in late March 2020, compared to the same time the year before, while online sales boomed - rising by $500 \%$ in late April (Nielson, 2020).

In terms of sleep duration, survey data from the Chinese general public in the February 2020 COVID-19 outbreak found that at least $20 \%$ met criteria for clinical insomnia, and the same proportion spent more than one hour awake in bed (Lin et al., 2020). Moreover, females, young people, and those who at greater risk for SARS-COV-2 exposure during the outbreak (e.g., first-line hospital workers) had more severe insomnia (Lin et al., 2020). During strict lockdown measures, those with psychiatric disorders prior to the pandemic were at even greater risk for elevated depressive and anxiety symptoms as well as insomnia (Hao et al., 2020). Insomnia is such a central problem during the pandemic that the European Cognitive-Behavioral Therapy Academy task force released practical recommendations for dealing with sleep problems during at-home confinement (Altena et al., 2020). The task force recommended using social media to share anxieties as well as positive "distractions," limiting exposure to COVID-19 news, and keeping a regular sleep-wake time, as well as other sleep hygiene tips.

Overeating is common during stressful times, particularly more energy-dense foods, thus fueling weight gain (Razzoli, Pearson, Crow, \& Bartolomucci, 2017). After one month of enforced lockdown in Northern Italy, obese individuals reported gaining an average of 3.3 pounds, which was associated with unhealthy food consumption, increased boredom, higher anxiety and depressive symptoms, and lower exercise (Pellegrini et al., 2020). There are also fears that pandemic-related school closures will fuel poor eating behavior and weight gain in children (Rundle, Park, Herbstman, Kinsey, \& Wang, 2020). Additionally, undernourishment, common among older adults, may jeopardize the elderly's vaccine response. 
Concerning physical activity, the World Health Organization recommends 150 minutes per week of moderate-intensity exercise like walking or 75 minutes a week of vigorous-intensity exercise like jogging. Achieving these guidelines is associated with a $17 \%$ lower risk of cardiovascular events, a $23 \%$ lower risk of cardiovascular mortality, and a $26 \%$ lower occurrence of type 2 diabetes (Wahid et al., 2016). These outcomes are relevant to COVID-19 patients, who are at risk for cardiovascular morbidity and mortality (Wahid et al., 2016). The American company Fitbit just released data from 30 million users that show $7 \%-38 \%$ reductions in average step counts across most countries in late March (Fitbit, 2020), but more peer-reviewed empirical evidence is needed.

Taken together, the COVID-19 pandemic and related stress promote poor health behaviors that, in turn worsen mental and physical health in a vicious cycle, ultimately driving weight gain. Ironically, the pandemic lifestyle could lower the efficacy of a SARS-CoV-2 vaccine.

\section{Treatment}

Intervention research corroborates the observed links between psychological and behavioral factors and vaccine responses discussed above. Specifically, these findings indicate that psychological and behavioral interventions may boost immune responses to vaccines. Interventions vary in type, dose, and duration, and thus they can be selected based on individual needs.

Psychological interventions as possible vaccine adjuvants include massage, meditation/mindfulness, expressive writing, and stress management (Vedhara et al., 2019). However, results are inconsistent likely due to sample age differences, vaccine type, intervention type, and varying times between vaccination and intervention, with improved antibody responses observed in 4 out of 7 randomized controlled trials (Vedhara et al., 2019). The median successful intervention length was 6 sessions and a total of 280 minutes (Vedhara et al., 2019). In particular, an 8-week cognitive-behavioral stress-management intervention among elderly spousal dementia caregivers enhanced antibody responses to influenza virus 
vaccination (Vedhara et al., 2003); 50\% of caregivers who received the intervention responded to vaccination, while only $7 \%$ and $29 \%$ of caregivers and non-caregiving controls not in the intervention responded, respectively. The mechanism remains unclear because caregivers maintained higher levels of distress throughout the intervention, compared to controls, and there were no between-group differences in cortisol. Similarly, in a randomized, controlled trial, an 8week mindfulness intervention among healthy employees in a work environment boosted antibody responses to an influenza vaccine between 4 and 8 weeks post-vaccination (Davidson et al., 2003). Expressive writing has mixed effects: short writing bouts about personal experiences of racism lowered antibody slopes by $40 \%$ to $50 \%$ (Stetler, Chen, \& Miller, 2006), while brief writing sessions about a traumatic event increased antibody levels over time (Petrie, Booth, Pennebaker, Davison, \& Thomas, 1995). Overall, randomized, controlled trials of psychological interventions to boost vaccine efficacy are few and far between, and further work is needed in this domain. In particular, psychological interventions that reliably reduce anxiety and depressive symptoms, such as a full course of cognitive-behavioral therapy, are worthy of further investigation as vaccine adjuvants. Additionally, more work is needed to see whether even brief psychological interventions reduce the prevalence and intensity of vaccine-related side effects.

Behavioral interventions have shown promising results. A systematic review on exercise interventions found fairly robust evidence that both short- and long-term exercise interventions can improve immune responses to vaccination, especially among those who are otherwise at risk for poor responses (e.g., sedentary individuals) (Pascoe, Singh, \& Edwards, 2014). Notably, a 25-minute eccentric arm exercise protocol targeting the muscle at the site of injection performed six hours prior to an influenza virus vaccine improved antibody responses in women and cell-mediated responses in men, compared to those who rested quietly (Edwards et al., 2007). Nevertheless, further investigation suggested that such an acute exercise protocol may only impact cell-mediated and antibody vaccine responses when the control group has a poor 
response (Campbell et al., 2010), and therefore may not be necessary for vaccines that are highly efficacious or demographics that have robust responses. A similar brief (15-minute) exercise protocol reduced days of swelling, fever, and low appetite, compared to a resting condition, among adolescents who received an HPV vaccine (V. Lee, Booy, Skinner, Fong, \& Edwards, 2018). Among older adults, a 45-minute moderate-intensity resistance exercise session immediately prior to receiving the influenza vaccine reduced vaccine reactions, including pain, redness, or swelling at injection site or other symptom/illness, compared to a resting condition (Bohn-Goldbaum et al., 2020). In terms of longer exercise protocols, ten months of cardiovascular training among sedentary, community-dwelling older adults did not affect peak antibody levels three and six weeks post- influenza vaccination, but it did promote clinically significant antibody levels six-months post-vaccination, compared to flexibility and balance training (Woods et al., 2009).

Along with exercise, boosting nutritional status via supplementation may result in a better antibody response to vaccines, especially in older adults, who often struggle to meet recommended daily nutritional guidelines especially if they live alone. In one randomized, controlled trial, elderly individuals who received a complete liquid nutritional supplement containing vitamins and minerals, including antioxidants, had higher antibody levels one month after influenza virus vaccine administration, compared to those who received the placebo (Wouters-Wesseling et al., 2002). There is little evidence that micronutrient supplementation at the time of vaccination improves responses (Savy et al., 2009). Even so, a randomized, doubleblind, placebo-controlled trial among nursing home elderly revealed that zinc supplementation successful increased serum zinc levels and led to increased T cell multiplication (Barnett et al., 2016), which could bode well for vaccine responsiveness. Also, preclinical evidence suggests that Vitamin A supplementation may have a similar effect (Tan, Sande, Pufnock, Blattman, \& Greenberg, 2011). Taken together, there is promising evidence that even short duration psychological and behavioral interventions can modify the immune system's vaccine response 
as well as vaccine-related side effects, but further research is needed to assess (1) the optimal dose and timing of the intervention, and (2) whether change in the targeted psychological construct or behavior is the mechanism driving the improved vaccine response.

\section{Other Virus-Related Considerations}

Psychological and behavioral factors interact with the current pandemic in many ways beyond vaccine response. These factors can influence susceptibility to infection upon SARSCoV-2 exposure and willingness to be vaccinated (i.e., vaccine uptake). The psychological vulnerabilities for infection upon virus exposure are elegantly reviewed elsewhere (Cohen, 2020), and largely overlap with vulnerabilities for poor vaccine response. Vaccine uptake is also a concern: recent data indicate that around half of Americans may refuse to receive a SARSCoV-2 vaccine (Cornwall, 2020). Although vaccine uptake is beyond the scope of this review, psychological factors clearly play a role at this stage as well.

\section{Conclusions}

Work from our lab and many others has identified psychological and behavioral factors as key determinants of the immune system's response to many different types of vaccines, helping to determine the side effects profile as well as efficacy. Unfortunately, the COVID-19 pandemic itself has aggravated many of the risk factors for poor vaccine responses, such as stress and sedentariness, although there is mixed evidence on loneliness, with one study finding stable levels, perhaps indicating some resilience. Overall, these risk factors are so prevalent that if not addressed, they could significantly reduce the overall efficacy of SARSCOV-2 vaccine candidates. Prior research suggests that psychological and behavioral interventions can improve vaccine responsiveness. Even shorter-term interventions can be effective. Therefore, now is the time to identify those at risk for a poor immune response and intervene on these risk factors. 


\section{References}

Afsar, B., Elsurer, R., Eyileten, T., Yilmaz, M. I., \& Caglar, K. (2009). Antibody response following hepatitis B vaccination in dialysis patients: Does depression and life quality matter? Vaccine, 27(42), 5865-5869.

Altena, E., Baglioni, C., Espie, C. A., Ellis, J., Gavriloff, D., Holzinger, B., ... Riemann, D. (2020). Dealing with sleep problems during home confinement due to the COVID-19 outbreak: Practical recommendations from a task force of the European CBT-I Academy. Journal of Sleep Research, e13052.

Bajema, K. L., Wiegand, R. E., Cuffe, K., Patel, S. V., lachan, R., Lim, T., ... Harding, L. (2020). Estimated SARS-CoV-2 seroprevalence in the US as of September 2020. JAMA Internal Medicine.

Barnett, J. B., Dao, M. C., Hamer, D. H., Kandel, R., Brandeis, G., Wu, D., ... Kong, E. (2016). Effect of zinc supplementation on serum zinc concentration and $\mathrm{T}$ cell proliferation in nursing home elderly: A randomized, double-blind, placebo-controlled trial. The American Journal of Clinical Nutrition, 103(3), 942-951.

Bohn-Goldbaum, E., Pascoe, A., Singh, M. F., Singh, N., Kok, J., Dwyer, D. E., ... Edwards, K. M. (2020). Acute exercise decreases vaccine reactions following influenza vaccination among older adults. Brain, Behavior, \& Immunity-Health, 1, 100009.

Brydon, L., Walker, C., Wawrzyniak, A. J., Chart, H., \& Steptoe, A. (2009). Dispositional optimism and stress-induced changes in immunity and negative mood. Brain, Behavior, and Immunity, 23(6), 810-816.

Brydon, L., Walker, C., Wawrzyniak, A., Whitehead, D., Okamura, H., Yajima, J., ... Steptoe, A. (2009). Synergistic effects of psychological and immune stressors on inflammatory cytokine and sickness responses in humans. Brain, Behavior, and Immunity, 23(2), 217224. 
Burns, V. E., Drayson, M., Ring, C., \& Carroll, D. (2002). Perceived stress and psychological well-being are associated with antibody status after meningitis $C$ conjugate vaccination. Psychosomatic Medicine, 64(6), 963-970.

Butler, M. J., \& Barrientos, R. M. (2020). The impact of nutrition on COVID-19 susceptibility and long-term consequences. Brain, Behavior, and Immunity, 87, 53-54.

Campbell, J. P., Edwards, K. M., Ring, C., Drayson, M. T., Bosch, J. A., Inskip, A., ... Burns, V. E. (2010). The effects of vaccine timing on the efficacy of an acute eccentric exercise intervention on the immune response to an influenza vaccine in young adults. Brain, Behavior, and Immunity, 24(2), 236-242.

Carstensen, L. L. (1992). Social and emotional patterns in adulthood: Support for socioemotional selectivity theory. Psychology and Aging, 7(3), 331.

Choon Lim Wong, G., Narang, V., Lu, Y., Camous, X., Nyunt, M. S. Z., Carre, C., ... Chua, M. (2019). Hallmarks of improved immunological responses in the vaccination of more physically active elderly females. Exercise Immunology Review, 25.

Christ, A., Lauterbach, M., \& Latz, E. (2019). Western diet and the immune system: An inflammatory connection. Immunity, 51(5), 794-811.

Clay, J. M., \& Parker, M. O. (2020). Alcohol use and misuse during the COVID-19 pandemic: A potential public health crisis? The Lancet Public Health, 5(5), e259.

Cohen, S. (2020). Psychosocial Vulnerabilities to Upper Respiratory Infectious Illness: Implications for Susceptibility to Coronavirus Disease 2019 (COVID-19). Perspectives on Psychological Science.

Cohen, S., Miller, G. E., \& Rabin, B. S. (2001). Psychological stress and antibody response to immunization: A critical review of the human literature. Psychosomatic Medicine, 63(1), 7-18. 
Cohen, S., Tyrrell, D., Russell, M. A., Jarvis, M. J., \& Smith, A. P. (1993). Smoking, alcohol consumption, and susceptibility to the common cold. American Journal of Public Health, 83(9), 1277-1283.

Cornwall, W. (2020, June 30). Just 50\% of Americans plan to get a COVID-19 vaccine. Here's how to win over the rest. Science Mag. Retrieved from https://www.sciencemag.org/news/2020/06/just-50-americans-plan-get-covid-19vaccine-here-s-how-win-over-rest

Dan, J. M., Mateus, J., Kato, Y., Hastie, K. M., Faliti, C., Ramirez, S. I., ... Rawlings, S. A. (2020). Immunological memory to SARS-CoV-2 assessed for greater than six months after infection. BioRxiv.

David, L. A., Maurice, C. F., Carmody, R. N., Gootenberg, D. B., Button, J. E., Wolfe, B. E., ... Fischbach, M. A. (2014). Diet rapidly and reproducibly alters the human gut microbiome. Nature, 505(7484), 559.

Davidson, R. J., Kabat-Zinn, J., Schumacher, J., Rosenkranz, M., Muller, D., Santorelli, S. F., ... Sheridan, J. F. (2003). Alterations in brain and immune function produced by mindfulness meditation. Psychosomatic Medicine, 65(4), 564-570.

Degos, F., Duhamel, G., Brechot, C., Nalpas, B., Courouce, A., Tron, F., \& Berthelot, P. (1986). Hepatitis B vaccination in chronic alcoholics. Journal of Hepatology, 2(3), 402-409.

Edwards, K. M., Burns, V. E., Allen, L. M., McPhee, J. S., Bosch, J. A., Carroll, D., ... Ring, C. (2007). Eccentric exercise as an adjuvant to influenza vaccination in humans. Brain, Behavior, and Immunity, 21(2), 209-217.

Elmer, T., Mepham, K., \& Stadtfeld, C. (2020). Students under lockdown: Comparisons of students' social networks and mental health before and during the COVID-19 crisis in Switzerland. Plos One, 15(7), e0236337. 
Fitzpatrick, K. M., Harris, C., \& Drawve, G. (2020). Living in the midst of fear: Depressive symptomatology among US adults during the COVID-19 pandemic. Depression and Anxiety, 37(10), 957-964.

Franceschi, C., Bonafè, M., Valensin, S., Olivieri, F., De Luca, M., Ottaviani, E., \& De Benedictis, G. (2000). Inflamm-aging: An evolutionary perspective on immunosenescence. Annals of the New York Academy of Sciences, 908(1), 244-254.

Gallagher, S., Phillips, A. C., Drayson, M. T., \& Carroll, D. (2009a). Caregiving for children with developmental disabilities is associated with a poor antibody response to influenza vaccination. Psychosomatic Medicine, 71(3), 341-344.

Gallagher, S., Phillips, A. C., Drayson, M. T., \& Carroll, D. (2009b). Parental caregivers of children with developmental disabilities mount a poor antibody response to pneumococcal vaccination. Brain, Behavior, and Immunity, 23(3), 338-346.

Gallagher, S., Phillips, A. C., Ferraro, A. J., Drayson, M. T., \& Carroll, D. (2008). Psychosocial factors are associated with the antibody response to both thymus-dependent and thymus-independent vaccines. Brain, Behavior, and Immunity, 22(4), 456-460.

Glaser, R., \& Kiecolt-Glaser, J. K. (2005). Stress-induced immune dysfunction: Implications for health. Nature Reviews Immunology, 5(3), 243.

Glaser, R., Kiecolt-Glaser, J. K., Bonneau, R. H., Malarkey, W., Kennedy, S., \& Hughes, J. (1992). Stress-induced modulation of the immune response to recombinant hepatitis B vaccine. Psychosomatic Medicine, 54(1), 22-29.

Glaser, R., Kiecolt-Glaser, J. K., Malarkey, W. B., \& Sheridan, J. F. (1998). The influence of psychological stress on the immune response to vaccines. Annals of the New York Academy of Sciences, 840(1), 649-655.

Glaser, R., Robles, T. F., Sheridan, J., Malarkey, W. B., \& Kiecolt-Glaser, J. K. (2003). Mild depressive symptoms are associated with amplified and prolonged inflammatory 
responses after influenza virus vaccination in older adults. Archives of General Psychiatry, 60(10), 1009-1014.

Glaser, R., Sheridan, J., Malarkey, W. B., MacCallum, R. C., \& Kiecolt-Glaser, J. K. (2000). Chronic stress modulates the immune response to a pneumococcal pneumonia vaccine. Psychosomatic Medicine, 62(6), 804-807.

Hao, F., Tan, W., Jiang, L., Zhang, L., Zhao, X., Zou, Y., ... Mclntyre, R. S. (2020). Do psychiatric patients experience more psychiatric symptoms during COVID-19 pandemic and lockdown? A case-control study with service and research implications for immunopsychiatry. Brain, Behavior, and Immunity, 87, 100-106.

Harris, V., Ali, A., Fuentes, S., Korpela, K., Kazi, M., Tate, J., ... de Weerth, C. (2018). Rotavirus vaccine response correlates with the infant gut microbiota composition in Pakistan. Gut Microbes, 9(2), 93-101.

Huang, Y., \& Zhao, N. (2020). Generalized anxiety disorder, depressive symptoms and sleep quality during COVID-19 outbreak in China: A web-based cross-sectional survey. Psychiatry Research, 112954.

Huda, M. N., Lewis, Z., Kalanetra, K. M., Rashid, M., Ahmad, S. M., Raqib, R., ... Stephensen, C. B. (2014). Stool microbiota and vaccine responses of infants. Pediatrics, 134(2), e362-e372.

Irwin, M. R., Levin, M. J., Laudenslager, M. L., Olmstead, R., Lucko, A., Lang, N., ... Weinberg, A. (2013). Varicella zoster virus-specific immune responses to a herpes zoster vaccine in elderly recipients with major depression and the impact of antidepressant medications. Clinical Infectious Diseases, 56(8), 1085-1093.

Jabaaij, L., Grosheide, P., Heijtink, R., Duivenvoorden, H., Ballieux, R., \& Vingerhoets, A. (1993). Influence of perceived psychological stress and distress on antibody response to low dose rDNA hepatitis B vaccine. Journal of Psychosomatic Research, 37(4), 361369. 
Jivraj, S., Nazroo, J., Vanhoutte, B., \& Chandola, T. (2014). Aging and subjective well-being in later life. Journals of Gerontology Series B: Psychological Sciences and Social Sciences, 69(6), 930-941.

Keylock, K. T., Lowder, T., Leifheit, K. A., Cook, M., Mariani, R. A., Ross, K., ... Woods, J. A. (2007). Higher antibody, but not cell-mediated, responses to vaccination in high physically fit elderly. Journal of Applied Physiology, 102(3), 1090-1098.

Kiecolt-Glaser, J. K., Derry, H. M., \& Fagundes, C. P. (2015). Inflammation: Depression fans the flames and feasts on the heat. American Journal of Psychiatry, 172(11), 1075-1091.

Kiecolt-Glaser, J. K., Glaser, R., Gravenstein, S., Malarkey, W. B., \& Sheridan, J. (1996). Chronic stress alters the immune response to influenza virus vaccine in older adults. Proceedings of the National Academy of Sciences, 93(7), 3043-3047.

Killgore, W. D., Cloonan, S. A., Taylor, E. C., Miller, M. M., \& Dailey, N. S. (2020). Three months of loneliness during the COVID-19 lockdown. Psychiatry Research, 113392.

Knoll, M. D., \& Wonodi, C. (2020). Oxford-AstraZeneca COVID-19 vaccine efficacy. The Lancet, S0140673620326234.

Kohut, M. L., Cooper, M. M., Nickolaus, M. S., Russell, D. R., \& Cunnick, J. E. (2002). Exercise and psychosocial factors modulate immunity to influenza vaccine in elderly individuals. The Journals of Gerontology Series A: Biological Sciences and Medical Sciences, 57(9), M557-M562.

Lang, P., Govind, S., Mitchell, W., Kenny, N., Lapenna, A., Pitts, D., \& Aspinall, R. (2010). Influenza vaccine effectiveness in aged individuals: The role played by cell-mediated immunity. European Geriatric Medicine, 1(4), 233-238.

Lange, T., Dimitrov, S., Bollinger, T., Diekelmann, S., \& Born, J. (2011). Sleep after vaccination boosts immunological memory. The Journal of Immunology, 187(1), 283-290.

Lange, T., Perras, B., Fehm, H. L., \& Born, J. (2003). Sleep enhances the human antibody response to hepatitis A vaccination. Psychosomatic Medicine, 65(5), 831-835. 
Lee, S. A., Jobe, M. C., Mathis, A. A., \& Gibbons, J. A. (2020). Incremental validity of coronaphobia: Coronavirus anxiety explains depression, generalized anxiety, and death anxiety. Journal of Anxiety Disorders, 102268.

Lee, V., Booy, R., Skinner, S., Fong, J., \& Edwards, K. (2018). The effect of exercise on local and systemic adverse reactions after vaccinations-Outcomes of two randomized controlled trials. Vaccine, 36(46), 6995-7002.

Li, J., Cowden, L. G., King, J. D., Briles, D. A., Schroeder Jr, H. W., Stevens, A. B., .. Wiener, H. W. (2007). Effects of chronic stress and interleukin-10 gene polymorphisms on antibody response to tetanus vaccine in family caregivers of patients with Alzheimer's disease. Psychosomatic Medicine, 69(6), 551-559.

Lin, L., Wang, J., Ou-yang, X., Miao, Q., Chen, R., Liang, F., ... Wang, T. (2020). The immediate impact of the 2019 novel coronavirus (COVID-19) outbreak on subjective sleep status. Sleep Medicine.

Luchetti, M., Lee, J. H., Aschwanden, D., Sesker, A., Strickhouser, J. E., Terracciano, A., \& Sutin, A. R. (2020). The trajectory of loneliness in response to COVID-19. American Psychologist, 75(7), 897-908.

Lynn, D. J., \& Pulendran, B. (2018). The potential of the microbiota to influence vaccine responses. Journal of Leukocyte Biology, 103(2), 225-231.

Mahase, E. (2020). Covid-19: Moderna vaccine is nearly 95\% effective, trial involving high risk and elderly people shows. BMJ: British Medical Journal (Online), 371.

Marsland, A. L., Cohen, S., Rabin, B. S., \& Manuck, S. B. (2001). Associations between stress, trait negative affect, acute immune reactivity, and antibody response to hepatitis $B$ injection in healthy young adults. Health Psychology, 20(1), 4.

Marsland, A. L., Cohen, S., Rabin, B. S., \& Manuck, S. B. (2006). Trait positive affect and antibody response to hepatitis B vaccination. Brain, Behavior, and Immunity, 20(3), 261269. 
Messaoudi, I., Asquith, M., Engelmann, F., Park, B., Brown, M., Rau, A., ... Grant, K. (2013). Moderate alcohol consumption enhances vaccine-induced responses in rhesus macaques. Vaccine, 32(1), 54-61.

Miller, G. E., Cohen, S., Pressman, S., Barkin, A., Rabin, B. S., \& Treanor, J. J. (2004).

Psychological stress and antibody response to influenza vaccination: When is the critical period for stress, and how does it get inside the body? Psychosomatic Medicine, 66(2), 215-223.

Morag, M., Morag, A., Reichenberg, A., Lerer, B., \& Yirmiya, R. (1999). Psychological variables as predictors of rubella antibody titers and fatigue-A prospective, double blind study. Journal of Psychiatric Research, 33(5), 389-395.

Oh, J. Z., Ravindran, R., Chassaing, B., Carvalho, F. A., Maddur, M. S., Bower, M., ... Yarovinsky, F. (2014). TLR5-mediated sensing of gut microbiota is necessary for antibody responses to seasonal influenza vaccination. Immunity, 41(3), 478-492.

Park, H.-L., Shim, S.-H., Lee, E.-Y., Cho, W., Park, S., Jeon, H.-J., .. Nam, J.-H. (2014). Obesity-induced chronic inflammation is associated with the reduced efficacy of influenza vaccine. Human Vaccines \& Immunotherapeutics, 10(5), 1181-1186.

Pascoe, A. R., Singh, M. A. F., \& Edwards, K. M. (2014). The effects of exercise on vaccination responses: A review of chronic and acute exercise interventions in humans. Brain, Behavior, and Immunity, 39, 33-41.

Pellegrini, M., Ponzo, V., Rosato, R., Scumaci, E., Goitre, I., Benso, A., ... Ghigo, E. (2020). Changes in weight and nutritional habits in adults with obesity during the "lockdown" period caused by the COVID-19 virus emergency. Nutrients, 12(7), 2016.

Petrie, K. J., Booth, R. J., Pennebaker, J. W., Davison, K. P., \& Thomas, M. G. (1995). Disclosure of trauma and immune response to a hepatitis $B$ vaccination program. Journal of Consulting and Clinical Psychology, 63(5), 787. 
Phillips, A. C., Carroll, D., Burns, V. E., \& Drayson, M. (2005). Neuroticism, cortisol reactivity, and antibody response to vaccination. Psychophysiology, 42(2), 232-238.

Phillips, A. C., Carroll, D., Burns, V. E., Ring, C., Macleod, J., \& Drayson, M. (2006). Bereavement and marriage are associated with antibody response to influenza vaccination in the elderly. Brain, Behavior, and Immunity, 20(3), 279-289.

Pietromonaco, P. R., \& Overall, N. C. (2020). Applying relationship science to evaluate how the COVID-19 pandemic may impact couples' relationships. American Psychologist.

Poland, G. A., Ovsyannikova, I. G., \& Kennedy, R. B. (2020). SARS-CoV-2 immunity: Review and applications to phase 3 vaccine candidates. The Lancet.

Prather, A. A., Hall, M., Fury, J. M., Ross, D. C., Muldoon, M. F., Cohen, S., \& Marsland, A. L. (2012). Sleep and antibody response to hepatitis B vaccination. Sleep, 35(8), 10631069.

Prendergast, A. J. (2015). Malnutrition and vaccination in developing countries. Philosophical Transactions of the Royal Society B: Biological Sciences, 370(1671), 20140141.

Pressman, S. D., Cohen, S., Miller, G. E., Barkin, A., Rabin, B. S., \& Treanor, J. J. (2005). Loneliness, social network size, and immune response to influenza vaccination in college freshmen. Health Psychology, 24(3), 297.

Raifman, M. A., \& Raifman, J. R. (2020). Disparities in the population at risk of severe illness from COVID-19 by race/ethnicity and income. American Journal of Preventive Medicine.

Razzoli, M., Pearson, C., Crow, S., \& Bartolomucci, A. (2017). Stress, overeating, and obesity: Insights from human studies and preclinical models. Neuroscience \& Biobehavioral Reviews, 76, 154-162.

Rebalancing the 'COVID-19 Effect' on Alcohol Sales. (2020, May 7). Nielson. Retrieved from https://www.nielsen.com/us/en/insights/article/2020/rebalancing-the-covid-19-effect-onalcohol-sales/ 
Reiter, P. L., Pennell, M. L., \& Katz, M. L. (2020). Acceptability of a COVID-19 vaccine among adults in the United States: How many people would get vaccinated? Vaccine, 38(42), $6500-6507$.

Rundle, A. G., Park, Y., Herbstman, J. B., Kinsey, E. W., \& Wang, Y. C. (2020). COVID-19Related School Closings and Risk of Weight Gain Among Children. Obesity, 28(6), 1006.

Savy, M., Edmond, K., Fine, P. E., Hall, A., Hennig, B. J., Moore, S. E., ... Prentice, A. M. (2009). Landscape analysis of interactions between nutrition and vaccine responses in children. The Journal of Nutrition, 139(11), 2154S-2218S.

Segerstrom, S. C., Hardy, J. K., Evans, D. R., \& Greenberg, R. N. (2012). Vulnerability, distress, and immune response to vaccination in older adults. Brain, Behavior, and Immunity, 26(5), 747-753.

Segerstrom, S. C., Schipper, L. J., \& Greenberg, R. N. (2008). Caregiving, repetitive thought, and immune response to vaccination in older adults. Brain, Behavior, and Immunity, 22(5), 744-752.

Smith, T. P., Kennedy, S. L., \& Fleshner, M. (2004). Influence of age and physical activity on the primary in vivo antibody and T cell-mediated responses in men. Journal of Applied Physiology, 97(2), 491-498.

Spiegel, K., Sheridan, J. F., \& Van Cauter, E. (2002). Effect of sleep deprivation on response to immunizaton. Jama, 288(12), 1471-1472.

Staff. (2020). The impact of coronavirus on global activity. Fitbit Blog, 25.

Stetler, C., Chen, E., \& Miller, G. E. (2006). Written disclosure of experiences with racial discrimination and antibody response to an influenza vaccine. International Journal of Behavioral Medicine, 13(1), 60-68.

Struve, J., Aronsson, B., Frenning, B., Granath, F., Sydow, M. V., \& Weiland, O. (1992). Intramuscular versus intradermal administration of a recombinant hepatitis B vaccine: A 
comparison of response rates and analysis of factors influencing the antibody response. Scandinavian Journal of Infectious Diseases, 24(4), 423-429.

Tan, W., Lu, Y., Zhang, J., Wang, J., Dan, Y., Tan, Z., ... Hu, Q. (2020). Viral kinetics and antibody responses in patients with COVID-19. MedRxiv.

Tan, X., Sande, J. L., Pufnock, J. S., Blattman, J. N., \& Greenberg, P. D. (2011). Retinoic acid as a vaccine adjuvant enhances CD8+ $T$ cell response and mucosal protection from viral challenge. Journal of Virology, 85(16), 8316-8327.

Twenge, J. M., \& Joiner, T. E. (2020). US Census Bureau-assessed prevalence of anxiety and depressive symptoms in 2019 and during the 2020 COVID-19 pandemic. Depression and Anxiety, 37(10), 954-956.

Vedhara, K., Ayling, K., Sunger, K., Caldwell, D. M., Halliday, V., Fairclough, L., ... Welton, N. J. (2019). Psychological interventions as vaccine adjuvants: A systematic review. Vaccine, 37(25), 3255-3266.

Vedhara, K., Bennett, P. D., Clark, S., Lightman, S. L., Shaw, S., Perks, P., ... Murphy, P. J. (2003). Enhancement of antibody responses to influenza vaccination in the elderly following a cognitive-behavioural stress management intervention. Psychotherapy and Psychosomatics, 72(5), 245-252.

Vedhara, K., Cox, N. K., Wilcock, G. K., Perks, P., Hunt, M., Anderson, S., ... Shanks, N. M. (1999). Chronic stress in elderly carers of dementia patients and antibody response to influenza vaccination. The Lancet, 353(9153), 627-631.

Vukmanovic-Stejic, M., Chambers, E. S., Suárez-Fariñas, M., Sandhu, D., Fuentes-Duculan, J., Patel, N., ... Larbi, A. (2018). Enhancement of cutaneous immunity during aging by blocking p38 mitogen-activated protein (MAP) kinase-induced inflammation. Journal of Allergy and Clinical Immunology, 142(3), 844-856.

Wahid, A., Manek, N., Nichols, M., Kelly, P., Foster, C., Webster, P., ... Rayner, M. (2016). Quantifying the association between physical activity and cardiovascular disease and 
diabetes: A systematic review and meta-analysis. Journal of the American Heart Association, 5(9), e002495.

Winter, A. P., Follett, E. A., Mclntyre, J., Stewart, J., \& Symington, I. S. (1994). Influence of smoking on immunological responses to hepatitis B vaccine. Vaccine, 12(9), 771-772.

Woods, J. A., Keylock, K. T., Lowder, T., Vieira, V. J., Zelkovich, W., Dumich, S., ... Cook, M. (2009). Cardiovascular exercise training extends influenza vaccine seroprotection in sedentary older adults: The immune function intervention trial. Journal of the American Geriatrics Society, 57(12), 2183-2191.

Wouters-Wesseling, W., Rozendaal, M., Snijder, M., Graus, Y., Rimmelzwaan, G., de Groot, L., \& Bindels, J. (2002). Effect of a complete nutritional supplement on antibody response to influenza vaccine in elderly people. The Journals of Gerontology Series A: Biological Sciences and Medical Sciences, 57(9), M563-M566.

Yang, S., Tian, G., Cui, Y., Ding, C., Deng, M., Yu, C., ... Li, Y. (2016). Factors influencing immunologic response to hepatitis B vaccine in adults. Scientific Reports, 6(1), 1-12.

Younas, M., Carrat, F., Desaint, C., Launay, O., \& Corbeau, P. (2017). Immune activation, smoking, and vaccine response. Aids, 31(1), 171-173.

Zuo, J., Dowell, A., Pearce, H., Verma, K., Long, H., Begum, J., ... Stapley, L. (2020). Robust SARS-CoV-2-specific T-cell immunity is maintained at 6 months following primary infection. BioRxiv. 